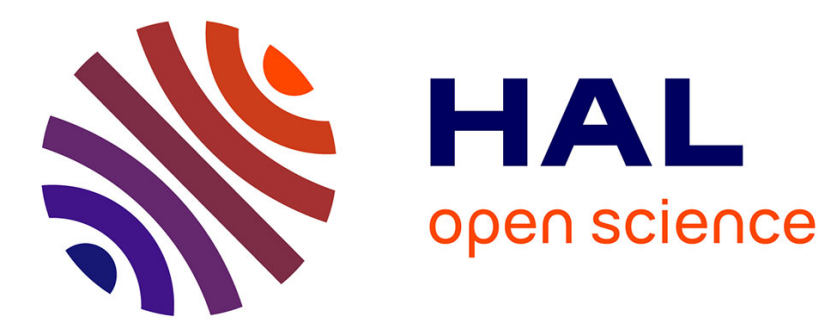

\title{
Modelling of sympathetic string vibrations in the clavichord using a modal Udwadia-Kalaba formulation
}

\author{
Jean-Théo Jiolat, Jean-Loïc Le Carrou, Jose Antunes, Christophe
} d'Alessandro

\section{- To cite this version:}

Jean-Théo Jiolat, Jean-Loïc Le Carrou, Jose Antunes, Christophe d'Alessandro. Modelling of sympathetic string vibrations in the clavichord using a modal Udwadia-Kalaba formulation. SEM. Proceedings of the 37th IMAC, A Conference and Exposition on Structural Dynamics 2019, 3, Springer, pp.277-280, 2019, Model Validation and Uncertainty Quantification. hal-02197017

\section{HAL Id: hal-02197017 \\ https://hal.science/hal-02197017}

Submitted on 29 Jul 2019

HAL is a multi-disciplinary open access archive for the deposit and dissemination of scientific research documents, whether they are published or not. The documents may come from teaching and research institutions in France or abroad, or from public or private research centers.
L'archive ouverte pluridisciplinaire HAL, est destinée au dépôt et à la diffusion de documents scientifiques de niveau recherche, publiés ou non, émanant des établissements d'enseignement et de recherche français ou étrangers, des laboratoires publics ou privés. 


\title{
Modelling of sympathetic string vibrations in the clavichord using a modal Udwadia-Kalaba formulation
}

\author{
J.T. Jiolat $^{1}$, J.L. Le Carrou ${ }^{1}$, J. Antunes ${ }^{2}$, C. d'Alessandro ${ }^{1}$ \\ ${ }^{1}$ Sorbonne Université, Centre National de la Recherche Scientifique, Institut Jean Le Rond d'Alembert, \\ 4, Place Jussieu 75252 Paris, France \\ ${ }^{2}$ Centro de Ciencias e Tecnologias Nucleares, Instituto Superior Técnico, Universidade de Lisboa, \\ Estrada Nacional 10, Km 139.7, Bobadela LRS, 2695-066, Portugal
}

\begin{abstract}
The vibratory and acoustic modeling of musical instruments is important for several purposes in cultural heritage preservation, performance studies and musical creation. On the one hand, building a model helps understanding the key features of an instrument, and then is useful for evaluation, documentation and preservation of historical models. On the other hand, modeling and simulation can help for improving existing instruments, or even designing new instruments by extension of the model. The clavichord is an early keyboard instrument equipped with a very simple mechanics. The strings are excited by small metal wedges or blades (the tangents) placed at the end of the keys. The tangent remains in contact with the strings for the duration of the note, defining the vibrating length of the string. All strings are coupled at a same bridge. A string is divided into three sections: a damped section (DS) between the hitch-pin and the tangent; the played section (PS), excited by the tangents, between the tangent and the bridge; and the resting section (RS) between the bridge and the tuning pin. Because of the coupling through the bridge of the PS and RS, the RS is set into vibration, acting as sympathetic strings. The vibratory responses of the RS is modelled using a modal approach based on the Udwadia-Kalaba formulation. Firstly, a review of the method is presented, accompanied with measurements performed on an instrument (copy of a Hubert 1784 fretted clavichord), which include an experimental modal analysis at the instrument bridge and measurements of string motions. Then, simulation results are reported and compared with experimental measurements.
\end{abstract}

Keywords: Sympathetic vibration, Clavichord, Udwadia-Kalaba formulation, String coupling, Modal analysis

\section{Introduction}

The sound of string instruments results of the vibratory behavior of coupled mechanical subsystems. These couplings can be studied by using physical modeling of several kinds. For instance, in the case of the concert harp, the coupling of the strings and the soundboard has been modeled by means of transfer matrices [3]. Also, it could be modeled by using finite element methods or experimental modal analysis, in particular using substructure techniques. In the case of the guitar, the couplings have been modeled by extracting the modal parameters of the soundboard at the bridge locations where the strings and the structure motions are coupled [1]. In the clavichord, a string is divided into three functional sections: a damped section (DS) between the hitch-pin and the tangent; the played section (PS), excited by the tangents, between the tangent and the bridge; and the resting section (RS) between the bridge and the tuning pin (see figure 1). The RS of the string is not directly excited by the tangent but is subjected to the motion constraint at the bridge. Then it is set into vibration, acting as sympathetic strings. Our objective is to predict the vibratory response of the RS of strings, set indirectly into vibration as a consequence of the excitation of one PS. To proceed accordingly, we first present the Udwadia-Kalaba (U-K) formulation and its modal extension, in order to compute the vibratory responses of a set of coupled mechanical substructures. Then, having extracted the necessary experimental modal parameters from our studied clavichord, we present some results from our numerical simulation. 


\section{Model U-K}

The U-K formulation was originally obtained from the Gauss principle of least action. Then, in the papers by Arabyan and Wu [2] and Laulusaand Bauchau [5], an original algebraic approach was found for deriving the U-K formulation for constrained systems from the classical formulation with Lagrange multipliers [1]. Let us consider a mechanical system with mass matrix $\mathbf{M}$ which is subjected to an external force vector $\mathbf{F}_{\mathbf{e}}(t)$, which includes all constraint-independent internal and external forces. This system is also subjected to a set of $P$ holonomic and non-holonomic constraints which depend on the system displacement $\mathbf{x}(t)$ and velocity $\mathbf{v}(t)$. Denoting the dynamical solution $\mathbf{x}_{\mathbf{u}}(t)$ of the unconstrained system and the one $\mathbf{x}(t)$ of the constrained system, which depends on the constraining forces $\mathbf{F}_{\mathbf{c}}(t)$, and following [2], one obtains the motion equations of the constrained system proposed by Udwadia and Kalaba [1,2] :

$$
\begin{gathered}
\ddot{\mathbf{x}}=\ddot{\mathbf{x}}_{u}+\mathbf{M}^{-1 / 2} \mathbf{B}^{+}\left(\mathbf{b}-\mathbf{A} \ddot{\mathbf{x}}_{\mathbf{u}}\right) . \\
\ddot{\mathbf{x}}_{u}=\mathbf{M}^{-1} \mathbf{F}_{e}(t)
\end{gathered}
$$

where $\mathbf{A}$ is the constraint matrix, $\mathbf{b}$ is a known constrained vector, $\mathbf{B}^{+}$is the Moore-Penrose inversion of matrix $\mathbf{B}=\mathbf{A} \mathbf{M}^{1 / 2}$. The original character of this approach is that it can be used for conservative or dissipative, linear or non-linear systems. Moreover, the generalized inverse $\mathbf{B}^{+}$can be rendered numerically robust, even when the constraint matrix is singular. For a particular excitation $\mathbf{F}_{e}(t)$, we can solve these equations using a suitable time-step integration scheme. Next, we adapt the U-K formulation in order to deal with continuous flexible systems whose dynamics will be described in terms of modal coordinates. We assume a set of $\mathrm{S}$ vibrating subsystems, each one defined in terms of its unconstrained modal basis and being coupled through $P$ kinematic constraints. Then, using the usual modal equations that govern the physical motion of the subsystems, we end up with similar equations of motion, which are described now in terms of modal parameters [1].

$$
\ddot{\mathbf{q}}=\boldsymbol{W} \tilde{\mathbf{M}}^{-1}\left(-\tilde{\mathbf{C}} \dot{\mathbf{q}}-\tilde{\mathbf{K}} \mathbf{q}+\mathbf{F}_{\mathbf{e x t}}\right)
$$

where $\mathbf{q}$ represent the vector of modal displacements, $\tilde{\mathbf{M}}, \tilde{\mathbf{K}}, \tilde{\mathbf{C}}$ are respectively the modal mass matrix, modal stiffness matrix, and modal damping matrix, while $\boldsymbol{W}=\mathbf{1}-\tilde{\mathbf{M}}^{-1 / 2} \boldsymbol{B}^{+} \boldsymbol{A}$ is a convenient global transformation matrix (which is computed before the time loop), where $\boldsymbol{A}$ is the modal constraint matrix, and $\mathbf{F}_{\text {ext }}$ are the external modal forces applied on the system. In order to proceed to the computation of the vibratory response of the constraint system, for a given external force vector, we need to obtain the modal parameters of each unconstrained subsystem. For the strings, we consider the classical mode shapes that we find theoretically for a flexible string. We also use a theoretical formulation for the damping of the string [4]. For the simulation, we decide to take 50 modes for each strings, covering a frequency range up to $24.5 \mathrm{kHz}$. Concerning the modal parameters of the instrument soundboard, which were measured at the bridge, these were obtained through experimental modal identification, using 37 points for the discretization along the bridge. Once we measured the vibratory frequency response functions (between a reference location and each point of the bridge), we proceeded to the modal identification using a frequency-domain approach called LSRF (Least-squares rational function estimation method), implemented in Matlab [6]. This modal analysis was performed within a frequency band going from $40 \mathrm{~Hz}$ to $800 \mathrm{~Hz}$, leading to 12 identified modes.

\section{Results and conclusion}

To compare our model with experimental data, we used a vibrometer to measure the vibratory velocity of the RS of the C5 string, at two centimeters from the bridge, induced by the tangent excitation of PS of the F3 string (i.e. playing the F3 key), all the other strings being muffled. The vibratory response is only measured in the vertical polarization of the motion of the string, since the model developed gives the response in just one polarization of motion. Our first step was to model the F3 PS and the G4 and C5 RS being coupled with the bridge (see figure 1). We choose these two strings because their RS have harmonic frequency relations with the harmonics of the PS of the F3 string: therefore a significant vibratory coupling should be expected. We produced numerically a realistic string excitation such that the response of the played string was as close as possible to the experimental response. In figure 2, we compare the spectral response of the C5 RS given by the numerical simulation with the measured one. In both results, we see the fundamental frequency peak of the F3 string which is at 328 $\mathrm{Hz}$ and all its harmonics, which are the partials transmitted to the C5 RS by means of the coupling with the bridge. Also, we note the presence of the fundamental frequency peak of the C5 RS which is at $491 \mathrm{~Hz}$ and its harmonics, being present because of the impulse response given to all substructures by the tangent excitation. Figure 2 shows a good agreement between 
the numerical simulation and measurement. So with this simplified model, we can take account of much of the physics being involved despite of the complexity of this instrument. For example, the coupling of the string with the bridge is quite simplified in the model. However, some spectral components do not have the same spectral amplitudes. In particular, we see that the partial at $200 \mathrm{~Hz}$ is absent in the simulation. We conjecture that this frequency peak comes from a soundboard mode of the clavichord which was not taken into account in the model. As for the other partials, their lack of spectral energy is probably due to a lack of precision in the estimation of the damping of the strings, and/or from some inaccuracy of the simulated string excitation. To further improve the model, we should consider all the 74 sympathetic strings of the Hubert clavichord in our simulation, which implies much longer computations. However, repeating the same measurement with all strings being free, the vibratory response of the RS of the C5 string remains quite unchanged. So we may not need to consider all the strings in the model to obtain a better result. Also, to improve our results, we should proceed to a more precise study of the damping of the strings and of the excitation features, to have a better estimation of the spectral amplitude of each partial of the computed response.

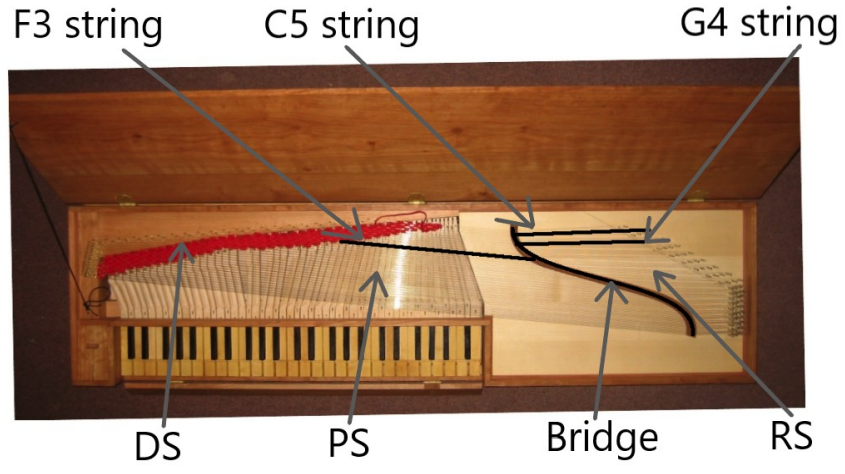

Fig. 1: Photo from above of the Hubert clavichord, with indications as to the substructures being modeled in our numerical simulations

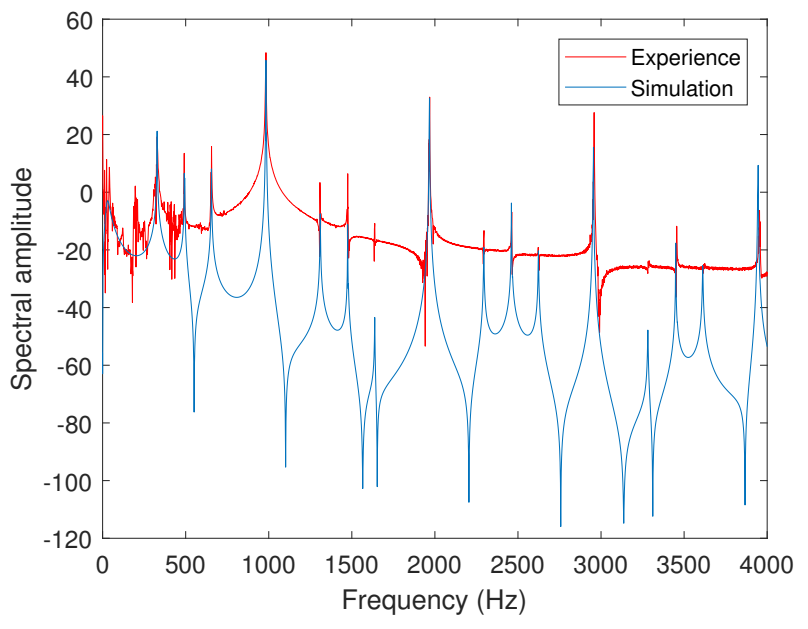

Fig. 2: Spectral comparison between the experimental signal measured with the vibrometer and the simulation of the C5 string having excited the F3 string of the Hubert clavichord

\section{References}

[1] Jose Antunes and Vincent Debut. Dynamical computation of constrained flexible systems using a modal udwadia-kalaba formulation: Application to musical instruments. The Journal of the Acoustical Society of America, 141(2):764-778, 2017.

[2] Ara Arabyan and Fei Wu. An improved formulation for constrained mechanical systems. Multibody System Dynamics, 2(1):49-69, 1998.

[3] Jean-Loïc Le Carrou, Francois Gautier, Nicolas Dauchez, and Joël Gilbert. Modelling of sympathetic string vibrations. Acta Acustica united with Acustica, 91(2):277-288, 2005.

[4] C Cuesta and C Valette. Mécanique de la corde vibrante. Paris: Hermes, 520, 1993.

[5] André Laulusa and Olivier A Bauchau. Review of classical approaches for constraint enforcement in multibody systems. Journal of Computational and Nonlinear Dynamics, 3(1):011004, 2008.

[6] Ahmet Arda Ozdemir and Suat Gumussoy. Transfer function estimation in system identification toolbox via vector fitting. IFAC-PapersOnLine, 50(1):6232-6237, 2017. 